(с) В.А. Петеркова ${ }^{1 *}$, А.С. Аметов ${ }^{2}$, А.Ю. Майоров ${ }^{1}$, Г.Р. Галстян ${ }^{1}$, Д.Н. Лаптев ${ }^{1}$, Н.А. Черникова ${ }^{2}$

${ }^{1}$ Национальный медицинский исследовательский центр эндокринологии, Москва

${ }^{2}$ Российская медицинская академия непрерывного профессионального образования, Москва

26 ноября 2020 г. состоялся Научно-консультативный совет под председательством академика РАН, профессора В.А. Петерковой, посвященный обсуждению возможности внедрения технологии непрерывного мониторинга глюкозы (НМГ) в клиническую практику врачей-эндокринологов в Российской Федерации с целью улучшения гликемического контроля у пациентов с сахарным диабетом (СД).

Основной задачей рабочей встречи было определение наиболее значимых с практической точки зрения показателей НМГ, необходимых для внедрения в клиническую практику, их характеристик для различных групп пациентов с СД.

В ходе мероприятия были обсуждены следующие темы и вопросы: важность выбора дополнительных критериев достижения гликемического контроля у пациентов с СД, помимо гликированного гемоглобина $\left(\mathrm{Hb}_{1 c}\right)$, место технологии НМГ в международных и российских клинических рекомендациях, точность систем НМГ и подходы к ее оценке, роль обучающих программ для пациентов с СД, в том числе обучения правильному использованию и интерпретации данных, получаемых при использовании устройств НМГ, анализ данных, полученных при изучении использования устройств НМГ в рандомизированных клинических исследованиях, а также данных, полученных в условиях повседневной клинической практики.

КЛЮЧЕВЫЕ СЛОВА: гликИрованный гемоглобин; непрерывный мониторинг глюкозы с периодическим сканированием; время в целевом диапазоне; flash-мониторинг глюкозы

\title{
THE SCIENTIFIC ADVISORY BOARD RESOLUTION: IMPLEMENTATION OF INTERMITTENTLY SCANNED CONTINUOUS GLUCOSE MONITORING IN CLINICAL PRACTICE TO IMPROVE GLYCEMIC CONTROL
}

( ) Valentina A. Peterkova ${ }^{*}$, Alexander S. Ametov², Aleksander Y. Mayorov', Gagik R. Galstyan', Dmitry N. Laptev', Natalya A. Chernikova²

${ }^{1}$ Endocrinology Research Centre, Moscow, Russia

${ }^{2}$ Russian Medical Academy of Continuous Professional Education, Moscow, Russia

The Scientific Advisory Board chaired by Academician of the Russian Academy of Sciences, Peterkova V.A. was held 26 of November in Moscow to discuss the possibilities of continuous glucose monitoring technology (CGM) implementation into routine clinical practice in Russia in order to improve glycemic control in patients with diabetes mellitus (DM).

The main aims for Advisory board were to determine the most significant indicators and parameters for CGM to be implemented in practice from a practical point of view of $\mathrm{LMWH}$, necessary for implementation in clinical practice, for different patients groups with diabetes.

The following questions and topics were raised within the discussion: the importance of additional indicators beyond glycated hemoglobin $\left(\mathrm{HbA}_{1 \mathrm{c}}\right)$ for glycemic control assessment in diabetes patients, CGM positioning in International and Russian clinical guidelines, the accuracy of CGM devises and approaches to its assessment, the role of education programs for diabetic patients, including trainings in correct use and data interpretation and analysis of CGM data obtained, clinical evidence analysis for CGM in randomized trials and real world evidence.

KEYWORDS: glycated hemoglobin; intermittently scanned continues glucose monitoring; time in range; flash monitoring 
26 ноября 2020 г. состоялся Научно-консультативный совет под председательством академика РАН, научного руководителя Института детской эндокринологии, заведующей кафедрой детской эндокринологии и диабетологии Института высшего и дополнительного профессионального образования ФГБУ «НМИЦ эндокринологии» Минздрава России, главного внештатного детского специалиста эндокринолога Минздрава России, профессора В.А. Петерковой, посвященный обсуждению возможности внедрения технологии непрерывного мониторинга глюкозы (НМГ) в клиническую практику врачей-эндокринологов в Российской Федерации с целью улучшения гликемического контроля у пациентов с сахарным диабетом (СД).

Основной задачей рабочей встречи было определение наиболее значимых с клинической точки зрения показателей непрерывного мониторинга глюкозы (НМГ), необходимых для внедрения в клиническую практику, их характеристик для различных групп пациентов с СД.

В своем докладе заведующий кафедрой эндокринологии ФГБОУ ДПО РМАНПО Минздрава России, профессор А.С. Аметов отметил, что достижение оптимального и стойкого контроля гликемии является одним из наиболее эффективных подходов для профилактики осложнений и снижения смертности у пациентов с СД. Несмотря на то что в настоящий момент гликированный гемоглобин ( $\left.\mathrm{HbA}_{1 с}\right)$ считается основным суррогатным маркером, позволяющим прогнозировать риск развития отдаленных осложнений СД, ограничением его использования является недостаток информации об острых эпизодах гипогликемии и гипергликемии, невозможность оценки их глубины и частоты; также по показателю $\mathrm{HbA}_{1 с}$ невозможно оценить вариабельность глюкозы в течение дня и между отдельными днями. Кроме того, такие состояния, как анемия, гемоглобинопатии, дефицит железа и бере- менность, могут искажать значения $\mathrm{HbA}_{1 c}$ [1]. В докладе профессора А.С. Аметова также были представлены данные, подтверждающие увеличение риска развития осложнений СД при высокой вариабельности гликемии [2].

В настоящий момент подходы к управлению СД должны включать не только контроль гликемии, но и минимизацию рисков развития гипогликемии, а также уменьшение вариабельности глюкозы. Вариабельность гликемии как терапевтическая мишень важна в лечении пациентов с СД 2 типа (СД2) [3-6]. В отличие от измерения $\mathrm{HbA}_{1 c^{\prime}}$ использование НМГ позволяет осуществлять непосредственное наблюдение за гликемическими экскурсиями и ежедневными профилями глюкозы, что может дать информацию для немедленных клинических решений и/или изменения образа жизни. НМГ также позволяет оценивать вариабельность глюкозы и выявлять закономерности гипогликемии и гипергликемии [1].

В 2019 г. был опубликован Консенсус по времени в целевом диапазоне, принятый на Конгрессе по передовым технологиям и лечению диабета (ATTD) (рис. 1) [1]. Согласованный список основных показателей НМГ был представлен 10 наиболее полезными в клинической практике показателями (табл. 1).

В рамках Консенсуса экспертная группа участников определила «время нахождения в диапазонах» как показатель гликемического контроля, который предоставляет более практическую информацию, чем $\mathrm{HbA}_{1 с}$. Основной целью эффективного и безопасного контроля глюкозы является увеличение времени в целевом диапазоне (ВЦД) при одновременном снижении времени ниже целевого диапазона (ВНД).

Доказана связь времени пребывания в целевом диапазоне с развитием микро- и макрососудистых осложнений СД. Так, риск развития микроальбуминурии у пациентов С СД 1 типа (СД1) возрастал на 40\% при уменьшении

\section{Сахарный диабет $1^{x}$ и 2 типа}

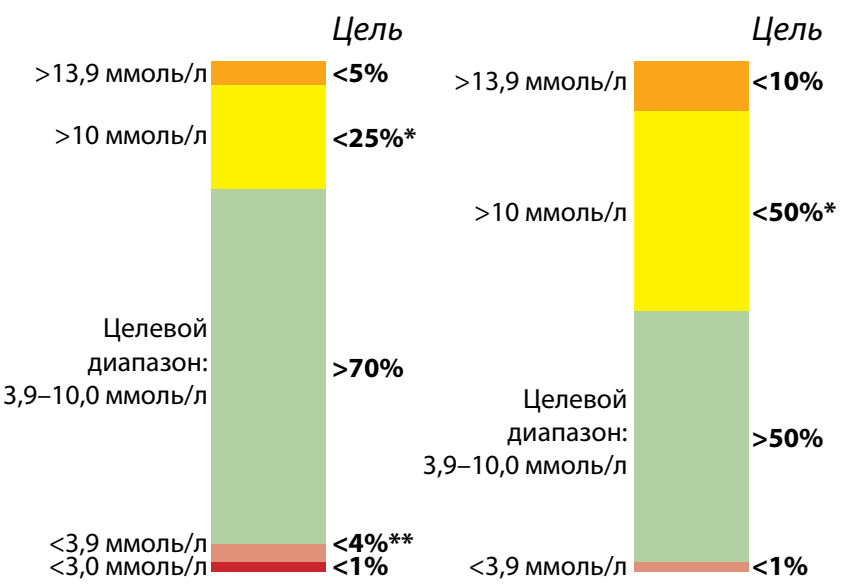

\section{Пожилые пациенты / с высоким риском: сахарный диабет 1 и 2 типа}

\section{Беременные: сахарный диабет 1 типа $^{\dagger}$}

\section{гестационный сахарный диабет и сахарный} диабет 2 типа ${ }^{5}$

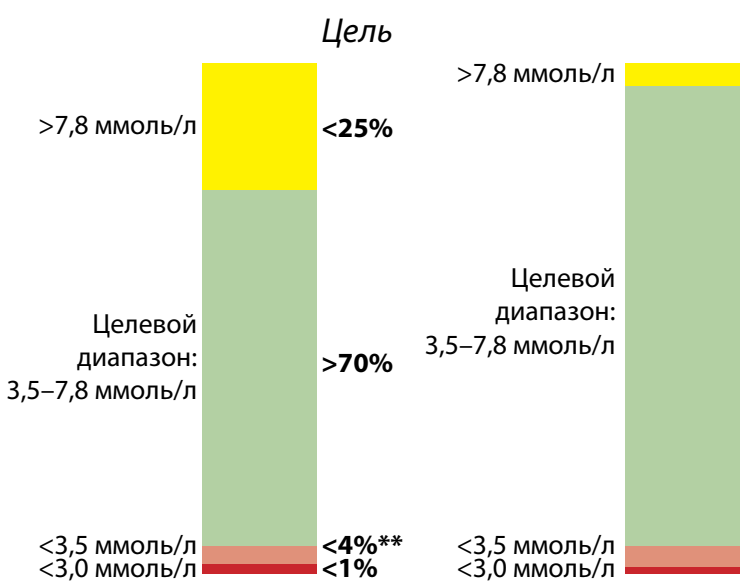

״Для возраста <25 лет при целевом уровне $\mathrm{HbA}_{1 c}$ 7,5\% ВЦД должно составлять примерно 60\%

†Приведенные проценты для времени нахождения в диапазонах основываются на ограниченных данных. Требуются дополнительные исследования. §роценты для времени нахождения в диапазонах не указаны в связи с чрезвычайно малым количеством данных. Требуются дополнительные исследования.

*Включая долю значений $>13,9$ ммоль/л

**Включая долю значений $<3,0$ ммоль/л

Рисунок 1. Целевые показатели при непрерывном мониторинге глюкозы для различных популяций пациентов с сахарным диабетом [1]. 
Таблица 1. Стандартизованные показатели непрерывного мониторинга глюкозы для клинического применения, 2019 г. [1]

1. Количество дней ношения устройства НМГ (рекомендовано 14 дней)

2. Доля времени с активным устройством НМГ ( $\geq 70 \%$ за 14 дней)

3. Среднее значение уровня глюкозы

4. Индикатор контроля уровня глюкозы (Glucose management indicator [GMI])

5. Целевые значения вариабельности глюкозы (\% CV) $\leq 36 \%$ (90)*

6. Время выше целевого диапазона (ВВД): доля измерений и время с гликемией >13,9 ммоль/л

Уровень 2

7. Время выше целевого диапазона (ВВД): доля измерений и время с гликемией 10,1-13,9 ммоль/л Уровень 1

8. Время в целевом диапазоне (ВЦД): доля измерений и время с гликемией 3,9-10,0 ммоль/л

Диапазон

9. Время ниже целевого диапазона (ВНД): доля измерений и время с гликемией 3,0-3,8 ммоль/л

Уровень 1

10. Время ниже целевого диапазона (ВНД): доля измерений и время с гликемией <3,0 ммоль/л

Уровень 2

Примечания: CV - коэффициент вариации. *Результаты отдельных исследований позволили предположить, что более низкие целевые значения KB (<33\%) обеспечивают дополнительную защиту от гипогликемии у пациентов, получающих инсулин или препараты сульфонилмочевины.

ВЦД на каждые 10\%, риск развития ретинопатии на 64\% при снижении ВЦД на каждые 10\% [7]. Пациенты с сахарным диабетом 2 типа (СД2) с прогрессирующими формами диабетической ретинопатии меньше времени находились в пределах целевого диапазона в сравнении с пациентами с легкими и умеренными формами диабетической ретинопатии [8]. Более низкие показатели ВЦД и высокая вариабельность глюкозы ассоциируются с более высокой частотой развития диабетической периферической нейропатии у пациентов с СД2 [9].

У пациентов с СД2 и кардиоваскулярными заболеваниями либо риском их возникновения показатели ВЦД $>70 \%$ ассоциируются с более низким риском развития серьезных сердечно-сосудистых нежелательных событий (major adverse cardiac events, MACE; отношение рисков (OP) 0,74; 95\% доверительный интервал (ДИ) 0,60-0,91; p<0,01) в сравнении с пациентами с ВЦД $\leq 70 \%$ [10].

В соответствии с рекомендациями Международного консенсуса по времени нахождения в диапазоне в качестве стандартизованного показателя для оценки вариабельности глюкозы следует применять коэффициент вариации (coefficient of variation - CV), показывающий, какой процент от среднего значения гликемии составляет среднее отклонение (CO) $[1,11]$. Высокая вариабельность глюкозы характеризуется значениями CV $\geq 36 \%$, а также ассоциируется с повышением частоты риска микро- и макрососудистых осложнений, а также с повышением риска возникновения гипогликемий [8].

В докладе заведующего отделом прогнозирования и инноваций диабета Института диабета, профессора кафедры диабетологии и диетологии Института высшего и дополнительного профессионального образования ФГБУ «НМИЦ эндокринологии» Минздрава России, Президента ОООИ «Российская Диабетическая Ассоциация» А.Ю. Майорова был представлен обзор материалов международных и Российских клинических рекоменда- ций с точки зрения применения технологии НМГ у пациентов с СД. В соответствии со Стандартами оказания медицинской помощи при СД Американской диабетической ассоциации (American Diabetes Association, ADA) в 2020 г., НМГ является ключевым фактором достижения гликемического контроля для большинства пациентов с СД1 и СД2. Применение технологии НМГ рекомендовано у пациентов с СД1, не достигших целей в контроле гликемии, с нарушением распознавания гипогликемии и/или с эпизодами гипогликемии для снижения уровня $\mathrm{HbA}_{1 c}$ и/или уменьшения гипогликемии. НМГ в сочетании с терапией инсулином является удобным инструментом для снижения уровня $\mathrm{HbA}_{1 с}$ и/или уменьшения гипогликемии у пациентов с СД2, не достигших целей в контроле гликемии. НМГ следует рассматривать как дополнительный инструмент для улучшения контроля глюкозы у всех детей и подростков с СД1 на фоне инсулинотерапии (инъекции, помпы), при этом польза от применения НМГ коррелирует с приверженностью пациента к его использованию [12].

В опубликованных в 2017 г. материалах Международного консенсуса по использованию НМГ рекомендуется применение устройств для НМГ у всех пациентов с СД1, а также у пациентов с СД2 (на интенсифицированной инсулинотерапии), не достигших целевых показателей гликемического контроля, в особенности у пациентов с проблемной гипогликемией [11].

В Клинических рекомендациях Международного общества по изучению диабета у детей и подростков (International Society for Pediatric and Adolescent Diabetes, ISPAD) указано, что применение НМГ у детей и подростков с СД может ассоциироваться с дополнительной пользой у пациентов с нарушением распознавания гипогликемии, а также с более значимым снижением $\mathrm{HbA}_{1 c}$ (в сравнении с традиционным самоконтролем глюкозы крови (СКГК)). При этом улучшение $\mathrm{HbA}_{1 c}$ коррелирует 
с количеством часов использования устройства НМГ в неделю [13].

В соответствии с Российскими клиническими рекомендациями по сахарному диабету, применение технологии НМГ у пациентов с СД1 рекомендовано для достижения индивидуальных целевых показателей гликемического контроля, снижения риска гипогликемии (в том числе тяжелой) и вариабельности гликемии, увеличения времени в целевом диапазоне, повышения качества жизни. У пациентов с СД2 на интенсифицированной инсулинотерапии применение НМГ рекомендуется для достижения индивидуальных целевых показателей гликемического контроля, снижения риска гипогликемии (в том числе тяжелой) и вариабельности гликемии, увеличения времени в целевом диапазоне, повышения качества жизни $[14,15]$.

В Алгоритмах специализированной медицинской помощи больным сахарным диабетом под редакцией И.И. Дедова, М.В. Шестаковой, А.Ю. Майорова указано, что использование НМГ может быть полезным для пациентов с СД любого типа, получающих интенсифицированную инсулинотерапию (многократные инъекции инсулина или инсулиновая помпа), исходно проводящих самоконтроль гликемии с частотой не менее 4 раз в сутки, особенно у беременных женщин, а также при нарушении распознавания гипогликемии [12]. Детям с СД1 рекомендуется применение НМГ с целью снижения $\mathrm{HbA}_{1 c^{\prime}}$ вариабельности гликемии, частоты гипогликемии [16].

В докладе профессора А.Ю. Майорова были представлены заключения профессиональных экспертных сообществ из ряда европейских стран, указывающих на возможность замещения устройствами НМГ рутинного СКГК, выполняемого при помощи глюкометров, а также рассмотрения НМГ в качестве предпочтительной опции для осуществления контроля глюкозы у пациентов с СД1 и СД2. В рекомендациях Национального института здоровья и клинического совершенствования Великобритании (National Institute for Health and Clinical Excellence, NICE) указано, что НМГ в терапии СД представляет собой в большинстве случаев альтернативу рутинному мониторингу уровня глюкозы в крови у людей с СД1 и СД2, которые используют инъекции инсулина [17]. Национальный консенсус по применению НМГ в Португалии рекомендует использование системы мониторинга глюкозы с периодическим сканированием (flash-мониторинга) для измерения уровня глюкозы в интерстиции пациентам с СД с 4-летнего возраста в качестве основного метода для осуществления самостоятельного контроля уровня глюкозы [18]. В соответствии с мнением экспертов-эндокринологов в Германии система мониторинга глюкозы с периодическим сканированием может быть использована для принятия клинических решений при СД, в том числе для коррекции дозы инсулина, следовательно, применение систем НМГ, в том числе НМГ с периодическим сканированием, способно заместить рутинную глюкометрию при помощи глюкометров, за исключением некоторых ситуаций (быстрые изменения уровня глюкозы, подтверждение гипогликемии и гипергликемии, в случаях несоответствия симптомов показаниям сканера) [19]. Профессор А.Ю. Майоров акцентировал внимание участников на том, что в настоящее время системы НМГ могут быть разделены на три категории: постоянный НМГ в «слепом» режиме, постоянный НМГ в реальном времени, а также НМГ с периодическим сканированием (flash-мониторинг), подчеркнув, что flash-мониторинг также относится к разновидности НMГ [12].

В своем выступлении, посвященном вопросам обучения и вовлеченности пациентов в процесс принятия решений и управления СД, профессор А.Ю. Майоров обратил внимание участников на то, что использование устройств НМГ необходимо сочетать с комплексным обучением пациентов, тренингами и поддержкой для оптимального внедрения данной технологии. В настоящее время устройства НМГ предоставляют данные не только об уровне глюкозы в настоящий момент, но и информацию о стрелках тенденций, которые указывают направление и скорость изменения уровня глюкозы - показателей, необходимых для коррекции дозы инсулина или дополнительного приема пищи. Одним из возможных подходов к коррекции дозы прандиального инсулина с учетом направления стрелки тенденций является подход, предложенный экспертами-эндокринологами из США. Данный подход основан на расчете дозы прандиального инсулина на основании фактора чувствительности к инсулину и направления стрелки тенденции с учетом актуального и целевого уровней значений глюкозы в интерстиции [20]. Разработка и проведение образовательных программ для пациентов с СД, находящихся на интенсифицированной инсулинотерапии, с использованием простых и понятных подходов к коррекции дозы инсулина на основании стрелок тенденций и принятие обоснованных клинических решений помогут пациентам улучшить контроль уровня глюкозы и достичь контроля над заболеванием [20].

Доцент кафедры эндокринологии, старший научный сотрудник отдела персонализированной медицины НИЦ ФГБОУ ДПО РМАНПО Минздрава России Н.А. Черникова представила данные о подходах к оценке точности систем НМГ на примере устройства для НМГ с периодическим сканированием. В соответствии с Консенсусом по НМГ 2017 г., в клинической практике следует использовать только те устройства, которые обеспечивают достаточный уровень точности измерений, однако к настоящему времени отсутствуют валидированные международные стандарты (International Organization for Standardization, ISO), а также Российские ГОСТы по оценке точности устройств НМГ $[11,21]$.

Одним из наиболее часто используемых показателей для оценки точности устройств для НМГ является средняя абсолютная относительная разница (Mean absolute relative difference [MARD]), характеризующая, насколько близки значения исследуемого датчика к референсным показателям. Однако данный показатель имеет ряд существенных особенностей, ограничивающих его широкое применение на практике: точность устройства НМГ при оценке по MARD зависит от числа парных измерений (показателей тестируемого датчика и референтного устройства - глюкометра либо определения глюкозы крови в условиях лаборатории) и действительно валидные значения MARD можно получить лишь при значительном числе парных измерений, что не всегда воспроизводимо; значения показателя MARD также находятся в зависимости от точности референтного 
устройства (например, точности глюкометра, если данное устройство было выбрано в качестве референтного); в связи с наличием физиологической задержки во времени между концентрацией глюкозы в крови и интерстиции, в особенности при быстрых изменениях уровня глюкозы крови, можно ожидать также различия в величинах MARD, измеренных при стабильном уровне глюкозы и при быстрых его изменениях даже при использовании одного и того же устройства НМГ $[21,22]$.

B связи с данными ограничениями показателя MARD, для оценки точности систем НМГ и возможности принятия клинических решений на основании показаний устройств НМГ, в настоящее время также используется анализ согласованной номограммы Паркс [21]. По степени влияния на клинические решения в номограмме Паркс выделяют несколько зон: измерения, попавшие в зону А, не оказывают негативного воздействия на клинические решения, принятые на основании измеренных данных. Результаты зоны В оказывают влияние на клиническое действие, но без эффекта либо с незначительным эффектом для клинического результата. Измерения, находящиеся в зонах C, D, E, оказывают влияние на клиническое действие и могут сопровождаться повышением риска возникновения негативных последствий при принятии решений, основанных на данных значениях [21, 23].

При исследовании сравнительной точности системы FreeStyle Libre у пациентов с СД1 и СД2 (n=72) в сравнении с показателями глюкозы крови, полученными при использовании встроенного в сканер глюкометра, было показано, что 99,7\% результатов датчика находятся в клинически приемлемых зонах А и В согласованной сетки ошибок (номограммы) Паркс, что позволяет принимать точные и безопасные для пациента клинические решения на основании данных датчика [24].

Заведующий отделением диабетической стопы Института диабета, профессор кафедры диабетологии и диетологии Института высшего и дополнительного профессионального образования ФГБУ «НМИЦ эндокринологии» Минздрава России Г.Р. Галстян представил данные рандомизированных контролируемых клинических исследований, наблюдательных исследований, а также результаты анализа данных, полученных в ходе реальной клинической практики, в которых проводилась оценка влияния НМГ с периодическим сканированием (flash-мониторинга глюкозы) на показатели гликемического контроля у взрослых пациентов с СД.

Так, в исследовании ІМРАСТ у взрослых пациентов с СД1 на фоне применения на протяжении 6 мес flash-мониторинга глюкозы отмечалось значимое снижение длительности и частоты гипогликемии (на $38 \%$ и $26 \%$ соответственно) по сравнению с контрольной группой, использовавшей СКГК ( $<<0,0001$ для обоих показателей). Уменьшение частоты и продолжительности гипогликемии не сопровождалось ростом $\mathrm{HbA}_{1 c^{\prime}}$ его значения оставались на уровне, соответствующем целевым значениям (<7,0\%). Применение flash-мониторинга глюкозы ассоциировалось с увеличением времени в целевом диапазоне и уменьшением частоты определения уровня глюкозы при помощи глюкометра (с 5,5 до 0,5 раза в день) [25].
В рандомизированном контролируемом исследовании по оценке влияния flash-мониторинга глюкозы на показатели гликемического контроля и качества жизни у пациентов с СД2 $(n=101)$ было показано, что в группе, использовавшей НМГ, снижение $\mathrm{HbA}_{1 с}$ через 10 нед составило 0,82\%, в группе СКГК - 0,33\% $(p=0,005)[26]$.

Улучшение гликемического контроля при оценке по уровню $\mathrm{HbA}_{1 c}$ на фоне использования flash-мониторинга глюкозы в течение 2-6 мес у взрослых пациентов с СД1 и СД2, а также у детей и подростков с СД1 было также подтверждено в ходе метаанализа, обобщившего результаты 25 исследований с общим числом пациентов, равным 2396. Применение системы НМГ с периодическим сканированием у пациентов с СД ассоциируется со снижением $\mathrm{HbA}_{1 с}$ в течение первых 2 мес с сохранением изменений на протяжении 12 мес. Среднее снижение $\mathrm{HbA}_{1 с}$ у взрослых с СД1 и СД2 на фоне использования НМГ составило 0,56\% (95\% ДИ -0,76--0,36). На каждый дополнительный процентный пункт выше 7,0\% в исходном $\mathrm{HbA}_{1 с}$ через 2-4 мес ожидается снижение $\mathrm{HbA}_{1 c}$ на 0,31\% (95\% ДИ -0,43--0,19) [27].

Для изучения влияния частоты сканирований при использовании системы flash-мониторинга глюкозы был проведен анализ обезличенных данных пациентов 7 европейских стран, полученных от 55343 сканеров и 64288 датчиков. Данные от сканеров были распределены в 20 групп в зависимости от частоты сканирований в сутки. Анализ данных показал, что при увеличении частоты сканирований отмечается снижение расчетного $\mathrm{HbA}_{1 c^{\prime}}$ времени в гипогликемии (<3,9 ммоль/л) на 15\%, увеличение времени пребывания в целевом диапазоне (3,9-10,0 ммоль/л) на 40\% в группе с наибольшей частотой сканирования в сравнении с группой данных с наименьшей частотой сканирования ( $p<0,001$ для всех показателей). Значимое уменьшение времени пребывания в гипогликемии отмечалось уже на 2-е сутки с начала использования и к 14-му дню достигало 13\% (уровень глюкозы <3,9 ммоль/л) и 23\% (уровень глюкозы <3,0 ммоль/л) в сравнении с исходными значениями ( $p<0,001$ для всех показателей) [28].

В результате анализа данных, полученных из регистра пациентов с СД1 и СД2 в Швеции (n=39 554), использование flash-мониторинга глюкозы в течение 12 мес ассоциировалось со стойким снижением $\mathrm{HbA}_{1 с}(-0,33 \%$ и -0,52\% соответственно, $p<0,0001)$ в сравнении с исходными показателями [29].

Ретроспективный анализ базы данных пациентов с СД1 $(n=33$ 203) и СД2 (n=40 955), предоставленных страховыми компаниями Франции, показал, что на фоне использования НМГ с периодическим сканированием отмечалось значимое уменьшение ежегодных госпитализаций по причине кетоацидоза у пациентов с СД1 и СД2 на 52\% и 47\% соответственно [30].

Заведующий детским отделением сахарного диабета Института детской эндокринологии, доцент кафедры детской эндокринологии-диабетологии Института высшего и дополнительного профессионального образования ФГБУ «НМИЦ эндокринологии» Минздрава России Д.Н. Лаптев в рамках доклада представил данные о влиянии НМГ с периодическим сканированием на показатели гликемического контроля у детей 
и подростков с СД с позиций доказательной медицины. В исследовании SELFY проводилась оценка влияния НМГ с периодическим сканированием на показатели гликемического контроля у детей и подростков в возрасте 4-17 лет $(n=76)$, страдающих СД1. Через 8 нед от начала использования системы flash-мониторинга глюкозы у пациентов отмечалось снижение $\mathrm{HbA}_{1 c}$ на $0,4 \%$ от исходного уровня $(\mathrm{p}<0,0001)$, время в целевом диапазоне возросло на 0,9 ч в сутки $(p=0,005)$, частота СКГК при помощи глюкометра сократилась

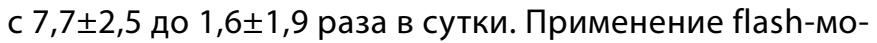
ниторинга глюкозы у подростков с СД1 и их родителей ассоциировалось с улучшением показателей общей удовлетворенности терапией при оценке по опроснику DTSQ в сравнении с исходными показателями $(p<0,0001)$ [31]. В результате проспективного наблюдательного исследования применения НМГ с периодическим сканированием у детей, подростков и молодых людей в возрасте 4-20 лет $(\mathrm{n}=334)$ с СД1 в условиях реальной практики было показано, что использование flash-мониторинга глюкозы в течение 12 мес позволяет уменьшить число эпизодов тяжелой гипогликемии на 53\% в сравнении с СКГК $(p=0,012)$ [32].

В докладе Д.Н. Лаптева были представлены данные об опыте применения системы НМГ с периодическим сканированием у 140 детей и подростков, наблюдавшихся в ФГБУ «НМИЦ эндокринологии» МЗ РФ, подтвердившие улучшение показателей гликемического контроля $\left(\mathrm{HbA}_{1 c^{\prime}}\right.$ ВЦД) через 24 нед от начала применения flashмониторинга глюкозы, а также сокращение потребности в СКГК на фоне использования устройства НМГ (неопубликованные данные).

В ходе общей дискуссии председатель научно-консультативного совета, академик РАН В.А. Петеркова предложила экспертам обсудить вопросы, касающиеся оптимальной частоты сканирований в сутки, оптимальную продолжительность периода сбора данных, полученных при НМГ, для последующего их анализа. Эксперты пришли к заключению, что оптимальная частота сканирований в сутки определяется индивидуально для каждого пациента с СД, при этом у пациентов с СД1, а также у пациентов с СД2, получающих интенсифицированную инсулинотерапию, оптимальной следует считать частоту сканирования 15 раз в сутки и более. В ходе дискуссии эксперты подчеркнули, что оптимальной продолжительностью сбора данных датчиком устройства НМГ является 14 дней, при этом амбулаторный гликемический профиль целесообразно рассматривать в качестве стандартизированного отчета для анализа всех основных показателей профиля глюкозы.

Председателем был озвучен вопрос о возможности рассматривать НМГ с периодическим сканированием как технологию, позволяющую принимать терапевтические решения у пациентов с СД, на который все эксперты ответили утвердительно.

\section{ЗАКЛЮЧЕНИЕ}

С учетом представленных данных и обсуждения в ходе общей дискуссии, участники совещания пришли к следующим заключениям.
- Целесообразно расширить перечень основных показателей, характеризующих достижение гликемического контроля, такими индикаторами, как целевой диапазон, ВЦД, ВНД и ВВД для различных групп пациентов с СД: пациентов молодого и среднего возраста без факторов риска, пожилых пациентов и пациентов с наличием факторов риска, беременных женщин, и включить данные показатели В Алгоритмы специализированной медицинской помощи больным сахарным диабетом и Российские клинические рекомендации по сахарному диабету. В качестве референсных значений для каждого из индикаторов необходимо рассматривать значения показателей, представленных в Консенсусе по времени в целевом диапазоне (табл. 1, рис. 1).

- К группам пациентов, применение технологии flash-мониторинга глюкозы у которых будет иметь дополнительные клинические преимущества, следует отнести:

взрослых пациентов с СД1 либо с СД2 на интенсифицированной инсулинотерапии, исходно проводящих самоконтроль гликемии с частотой не менее 4 раз в сутки, беременных женщин, пациентов, не достигших целевых значений $\mathrm{HbA}_{1 c^{\prime}}$ а также пациентов с нарушением распознавания гипогликемии для достижения индивидуальных целевых показателей гликемического контроля, снижения риска гипогликемии (в том числе тяжелой) и вариабельности гликемии, увеличения времени в целевом диапазоне, повышения качества жизни;

детей до 18 лет с СД1 с НbA 1 выше индивидуального целевого показателя, а также независимо от уровня $\mathrm{HbA}_{1 c}$ при: наличии тяжелых гипогликемий ( $\geq 1$ раза за последний год), частых эпизодах легкой гипогликемии, нарушении распознавания гипогликемии, высокой вариабельности гликемии.

- Необходимы разработка и внедрение обучающих программ для врачей и пациентов по использованию устройств НМГ, а также по интерпретации данных, полученных при НМГ.

- Технология НМГ с периодическим сканированием (flash-мониторинг) может использоваться для принятия клинических решений у пациентов с СД, в том числе для коррекции дозы инсулина.

- Целесообразно внесение в Алгоритмы специализированной медицинской помощи больным сахарным диабетом, а также в Российские клинические рекомендации по сахарному диабету положения о НМГ в следующей редакции: «У пациентов с СД1 и СД2 в качестве метода для осуществления контроля уровня глюкозы могут использоваться самоконтроль при помощи глюкометров и/или устройства для НМГ».

\section{ДОПОЛНИТЕЛЬНАЯ ИНФОРМАЦИЯ}

Источники финансирования. Данная статья является резюме Совета Экспертов, проведенного при поддержке компании Abbott.

Конфликт интересов. Все авторы статьи принимали участие в научно-консультативном совете по применению технологии 
непрерывного мониторинга глюкозы с периодическим сканированием в достижении гликемического контроля, который проводился при поддержке компании Abbott, результаты которого легли в основу этой статьи.

Финансирование работы. Научно-консультативный совет экспертов, результаты которого легли в основу этой статьи, проводился при поддержке комании Abbott. Первый вариант рукописи также был поддержан компанией Abbott.

Участие авторов. Петеркова В.А. - концепция, редактирование, финальное утверждение рукописи; Аметов А.С., Майоров А.Ю., Галстян Г.Р., Лаптев Д.Н., Черникова Н.А. - редактирование и финальное утверждение рукописи.

\section{СПИСОК ЛИТЕРАТУРЫ | REFERENCES}

1. Battelino T, Danne T, Bergenstal RM, et al. Clinical Targets for Continuous Glucose Monitoring Data Interpretation: Recommendations From the International Consensus on Time in Range. Diabetes Care. 2019;42(8):1593-1603. doi: https://doi.org/10.2337/dci19-0028

2. Monnier L, Colette C. Glycemic Variability: Should we and can we prevent it? Diabetes Care. 2008;31(2):S150-S154. doi: https://doi.org/10.2337/dc08-s241.

3. Rayman G. Glycaemic control, glucose variability and the Triangle of Diabetes Care. Br J Diabetes. 2016;16:3. doi: https://doi.org/10.15277/bjd.2016.070

4. Аметов А.С., Черникова Н.А., Демидова Т.Ю. Вариабельность гликемии как терапевтическая мишень в лечении пациентов с сахарным диабетом 2 типа // Фарматека. — 2016. — №5. C. 8-13. [Ametov AS, Chernikova NA, Demidova TYu. Glycemic variability as a therapeutic target in the treatment of patients with type 2 diabetes mellitus. Farmateka. 2016;5:8-13. (In Russ.)].

5. Тиселько А.В., Ярмолинская М.И., Мишарина Е.В. Оценка вариабельности гликемического профиля как основа стратегии инсулинотерапии у беременных с сахарным диабетом 1 типа // Сахарный диабет. - 2019. - Т. 22. - №6. - С. 526-535. [Tiselko AV, Yarmolinskaya MI, Misharina EV. Evaluation of glycaemic profile variability as a basis for insulin therapy strategy in pregnant women with type 1 diabetes. Diabetes Mellitus. 2020;22(6):526-535. (In Russ.)]. doi: https://doi.org/10.14341/DM10214

6. Лаптев Д.Н. Связь гипогликемии и вариабельности гликемии с автономной дисфункцией у детей и подростков с сахарным диабетом 1 типа // Сахарный диабет. — 2014. - Т. 17. — №4. C. 87-92. [Laptev DN. Relationship of hypoglycemia and glucose variability with autonomic dysfunction in children and adolescents with type 1 diabetes. Diabetes Mellitus. 2014;17(4):87-92. (In Russ.)]. doi: https://doi.org/10.14341/DM2014487-92

7. Beck RW, Bergenstal RM, Riddlesworth TD, et al. Validation of Time in Range as an Outcome Measure for Diabetes Clinical Trials. Diabetes Care. 2019;42(3):400-405. doi: https://doi.org/10.2337/dc18-1444

8. Lu J, Ma X, Zhou J, et al. Association of Time in Range, as Assessed by Continuous Glucose Monitoring, With Diabetic Retinopathy in Type 2 Diabetes. Diabetes Care. 2018;41(11):2370-2376. doi: https://doi.org/10.2337/dc18-1131

9. Mayeda L, Katz R, Ahmad I, et al. Glucose time in range and peripheral neuropathy in type 2 diabetes mellitus and chronic kidney disease. BMJ Open Diabetes Res Care. 2020;8(1):e000991. doi: https://doi.org/10.1136/bmjdrc-2019-000991

10. Bergenstal $\mathrm{R}$, Hachmann-Nielsen E, Kvist K. Derived time-in-range is associated with MACE in type 2 diabetes: data from the DEVOTE trial. Presented at EASD. 2020. Oral Presentation. $159 \mathrm{p}$

11. Danne T, Nimri R, Battelino T, et al. International Consensus on Use of Continuous Glucose Monitoring. Diabetes Care. 2017;40(12):1631-1640. doi: https://doi.org/10.2337/dc17-1600

12. Дедов И.И., Шестакова М.В., Майоров А.Ю. и др. Алгоритмы специализированной медицинской помощи больным сахарным диабетом: Клинические рекомендации (Вып. 9) // Сахарный диабет. - 2019. - T. 22. — №S1. - C. 1-144. [Dedov II, Shestakova MV, Mayorov AYu, et al. Standards of specialized diabetes care. Diabetes Mellitus. 2019;22(S1):1-144 (In Russ.)]. doi: https://doi.org/10.14341/DM221S1

13. DiMeglio LA, Acerini CL, Codner E, et al. ISPAD Clinical Practice Consensus Guidelines 2018: Glycemic control targets and glucose monitoring for children, adolescents, and young adults with diabetes. Pediatr Diabetes. 2018;19:105-114. doi: https://doi.org/10.1111/pedi.12737

14. Сахарный диабет 1 типа у взрослых. Клинические рекомендации // Сахарный диабет. - 2020. - Т. 23. №S1. - C. 42-114. [Clinical guidlines. Diabetes mellitus type 1 in adults. Diabetes Mellitus. 2020;23(S1):42-114. (In Russ.)]. doi: https://doi.org/10.14341/DM20201S
15. Сахарный диабет 2 типа у взрослых. Клинические рекомендации // Сахарный диабет. - 2020. - Т. 23. №S2. - C. 4-102. [Clinical guidlines. Diabetes mellitus type 2 in adults. Diabetes Mellitus. 2020;23(S2):4-102. (In Russ.)]. doi: https://doi.org/10.14341/DM20202S

16. Сахарный диабет 1 типа у детей. Клинические рекомендации Сахарный диабет. - 2020. - T. 23. - №S1. - C. 4-40. [Clinical guidlines. Diabetes mellitus type 1 in childhood. Diabetes mellitus type 1 in adults. Diabetes Mellitus. 2020;23(S1):4-40. (In Russ.)]. doi: https://doi.org/10.14341/DM20201S

17. National Institute for Health and Care Excellence (NICE). FreeStyle Libre for glucose monitoring (MIB1 10). 2017, July. P. 1-20.

18. Cardoso H, et al. Consenso Nacional para a Utilização do Sistema de Monitorização Flash da Glicose National Consensus on the Use of the Glucose Flash Monitoring System CONSENSO NACIONAL. Rev. Port. Diabetes. 2018;13(4):143-153.

19. Freckmann G, Schlüter S, Heinemann L. Replacement of Blood Glucose Measurements by Measurements With Systems for Real-Time Continuous Glucose Monitoring (rtCGM) or CGM With Intermittent Scanning (iscCGM): A German View. J Diabetes Sci Technol. 2017;11(4):653-656. doi: https://doi.org/10.1177/1932296817721004

20. Kudva YC, Ahmann AJ, Bergenstal RM, et al. Approach to Using Trend Arrows in the FreeStyle Libre Flash Glucose Monitoring Systems in Adults. J Endocr Soc. 2018;2(12):1320-1337. doi: https://doi.org/10.1210/js.2018-00294

21. Ajjan RA, Cummings $M H$, Jennings $P$, et al. Accuracy of flash glucose monitoring and continuous glucose monitoring technologies: Implications for clinical practice. Diabetes Vasc Dis Res. 2018;15(3):175-184. doi: https://doi.org/10.1177/1479164118756240

22. Reiterer F, Polterauer P, Schoemaker M, et al. Significance and Reliability of MARD for the Accuracy of CGM Systems. J Diabetes Sci Technol. 2017;11(1):59-67. doi: https://doi.org/10.1177/1932296816662047

23. Parkes JL, Slatin SL, Pardo S, Ginsberg BH. A new consensus error grid to evaluate the clinical significance of inaccuracies in the measurement of blood glucose. Diabetes Care. 2000;23(8):1143-1148. doi: https://doi.org/10.2337/diacare.23.8.1143

24. Bailey T, Bode BW, Christiansen MP, et al. The Performance and Usability of a Factory-Calibrated Flash Glucose Monitoring System. Diabetes Technol Ther. 2015;17(11):787-794. doi: https://doi.org/10.1089/dia.2014.0378

25. Bolinder J, Antuna R, Geelhoed-Duijvestijn P, Kröger J, Weitgasser R. Novel glucose-sensing technology and hypoglycaemia in type 1 diabetes: a multicentre, non-masked, randomised controlled trial. Lancet. 2016;388(10057):2254-2263. doi: https://doi.org/10.1016/S0140-6736(16)31535-5

26. Yaron M, Roitman E, Aharon-Hananel G, et al. Effect of Flash Glucose Monitoring Technology on Glycemic Control and Treatment Satisfaction in Patients With Type 2 Diabetes. Diabetes Care. 2019;42(7):1178-1184. doi: https://doi.org/10.2337/dc18-0166

27. Evans M, Welsh Z, Ells S, Seibold A. The Impact of Flash Glucose Monitoring on Glycaemic Control as Measured by HbA1c: A Meta-analysis of Clinical Trials and Real-World Observational Studies. Diabetes Ther. 2020;11(1):83-95. doi: https://doi.org/10.1007/s13300-019-00720-0

28. Dunn TC, Xu Y, Hayter G, Ajjan RA. Real-world flash glucose monitoring patterns and associations between self-monitoring frequency and glycaemic measures: A European analysis of over 60 million glucose tests. Diabetes Res Clin Pract. 2018;137:37-46. doi: https://doi.org/10.1016/j.diabres.2017.12.015 
29. Eeg-Olofsson K, Svensson A-M, Franzén S, et al. 74-LB: Sustainable $\mathrm{HbA1c}$ Decrease at 12 Months for Adults with Type 1 and Type 2 Diabetes Using the FreeStyle Libre System: A Study within the National Diabetes Register in Sweden. Diabetes. 2020;69(1):74-LB. doi: https://doi.org/10.2337/db20-74-LB.

30. Roussel R, Guerci B, Vicaut E, et al. 68-OR: Dramatic Drop in Ketoacidosis Rate after FreeStyle Libre System Initiation in Type 1 and Type 2 Diabetes in France, Especially in People with Low SelfMonitoring of Blood Glucose (SMBG): A Nationwide Study. Diabetes. 2020;69(1):68-OR. doi: https://doi.org/10.2337/db20-68-OR
31. Campbell FM, Murphy NP, Stewart C, et al. Outcomes of using flash glucose monitoring technology by children and young people with type 1 diabetes in a single arm study. Pediatr Diabetes. 2018;19(7):1294-1301 doi: https://doi.org/10.1111/pedi.12735

32. Messaaoui A, Tenoutasse S, Crenier L. Flash Glucose Monitoring Accepted in Daily Life of Children and Adolescents with Type 1 Diabetes and Reduction of Severe Hypoglycemia in Real-Life Use. Diabetes Technol Ther. 2019;21(6):329-335. doi: https://doi.org/10.1089/dia.2018.0339

\section{ИНФОРМАЦИЯ ОБ АВТОРАХ [AUTHORS INFO]}

*Петеркова Валентина Александровна, д.м.н., профессор, академик PAH [Valentina A. Peterkova, MD, PhD, Professor]; адрес: Россия, 117036, Москва, ул. Дм. Ульянова, д. 11 [address: 11 Dm. Ulyanova street, 117036 Moscow, Russia]; ORCID: https://orcid.org/0000-0002-5507-4627; eLibrary SPIN: 4009-2463; e-mail: peterkovava@hotmail.com

Аметов Александр Сергеевич, д.м.н., професcop [Alexander S. Ametov, MD, PhD, Professor];

ORCID: https://orcid.org/0000-0002-7936-7619; e-mail: alexander.ametov@gmail.com

Майоров Александр Юрьевич, д.м.н. [Aleksander Y. Mayorov, MD, PhD]; ORCID: https://orcid.org/0000-0001-5825-3287; eLibrary SPIN: 4275-7779; e-mail: education@endocrincentr.ru

Галстян Гагик Радикович, Д.М.Н., професcop [Gagik R. Galstyan, MD, PhD, Professor];

ORCID: https://orcid.org/0000-0001-6581-4521; eLibrary SPIN: 9815-7509; e-mail: galstyangagik964@gmail.com

Лаптев Дмитрий Никитич, д.м.H. [Dmitry N. Laptev, MD, PhD]; ORCID: https://orcid.org/0000-0002-4316-8546;

eLibrary SPIN: 2419-4019; e-mail: laptevdn@ya.ru

Черникова Наталья Альбертовна, к.M.н. [Natalya A. Chernikova, MD, PhD]; ORCID: https://orcid.org/0000-0002-0562-8396; e-mail: nachendoc@yandex.ru

\section{ЦИТИРОВАТЬ:}

Петеркова В.А., Аметов А.С., Майоров А.Ю., Галстян Г.Р., Лаптев Д.Н., Черникова Н.А. Резолюция научно-консультативного совета «Применение технологии непрерывного мониторинга глюкозы с периодическим сканированием в достижении гликемического контроля» // Сахарный диабет. — 2021. — Т. 24. — №2. — С. $185-192$. doi: https://doi.org/10.14341/DM12753

\section{TO CITE THIS ARTICLE:}

Peterkova VA, Ametov AS, Mayorov AY, Galstyan GR, Laptev DN, Chernikova NA. The Scientific Advisory board resolution: Implementation of intermittently scanned Continuous Glucose monitoring in clinical practice to improve glycemic control. Diabetes Mellitus. 2021;24(2):185-192. doi: https://doi.org/10.14341/DM12753 